Nota científica

\title{
Helmintos intestinales de Amazona amazonica (Psittaciformes: Psittacidae) de vida libre en la región noreste de Brasil
}

\author{
Intestinal helminths of wild Amazona amazonica (Psittaciformes: Psittacidae) in Northeastern Brazil \\ Elizandra Gomes-dos Santos ${ }^{a}$, Jaqueline Bianque-de Oliveira ${ }^{\text {b,* }}$, \\ Geraldo Jorge Barbosa-de Moura ${ }^{a}$ y Jozélia Maria de Souza-Correia ${ }^{a}$ \\ ${ }^{a}$ Laboratório de Estudos Herpetológicos e Paleoherpetológicos, Universidade Federal Rural de Pernambuco, Rua Dom Manoel de Medeiros, Dois Irmãos, \\ CEP 52171-900, Recife, Pernambuco, Brasil \\ b Laboratório de Parasitologia, Universidade Federal Rural de Pernambuco, Rua Dom Manoel de Medeiros, Dois Irmãos, CEP 52171-900, Recife, \\ Pernambuco, Brasil
}

Recibido el 29 de septiembre de 2014; aceptado el 21 de abril de 2015

Disponible en Internet el 21 de agosto de 2015

\section{Resumen}

Los helmintos, especialmente los gastrointestinales, son uno de los grupos de parásitos más comunes en las aves silvestres. El objetivo de este estudio fue identificar los parásitos gastrointestinales de Amazona amazonica (Linnaeus, 1766) del estado de Pernambuco, noreste de Brasil. Once de las 19 aves estaban parasitadas (11/19; 57.9\%): el nematodo Ascaridia hermaphrodita (Froelich, 1789) fue encontrado en 8 aves (8/11; 72.7\%) y el cestodo Raillietina sp. lo fue en 3 aves $(3 / 11 ; 27.3 \%)$, ambos registrados por primera vez en A. amazonica en el estado de Pernambuco.

Derechos Reservados (C) 2015 Universidad Nacional Autónoma de México, Instituto de Biología. Este es un artículo de acceso abierto distribuido bajo los términos de la Licencia Creative Commons CC BY-NC-ND 4.0.

Palabras clave: Loros; Ascaridia hermaphrodita; Raillietina; Conservación

\begin{abstract} both first recorded in A. amazonica from Pernambuco State. Creative Commons CC License BY-NC-ND 4.0.

Keywords: Parrots; Ascaridia hermaphrodita; Raillietina; Conservation

Las enfermedades infecciosas y parasitarias pueden afectar negativamente la biodiversidad (Catão-Dias, 2003). La presencia de parásitos es afectada, entre otros factores, por condiciones ambientales (naturales y antrópicas) y la disponibilidad de alimento (Costa, Coelho, Bueno, Ferreira y Freire, 2010). Los helmintos, especialmente los gastrointestinales, son uno de los
\end{abstract}

Helminths, especially gastrointestinal, are one of the most common parasites in wild birds. The aim of this study was to identify the gastrointestinal parasites of Amazona amazonica (Linnaeus, 1766) in Pernambuco State, Northeastern Brazil. Eleven of the 19birds were parasitized (11/19; 57.9\%): the nematode Ascaridia hermaphrodita (Froelich, 1789) was found in 8 birds (8/11; 72.7\%), and the cestode Raillietina sp. in 3 birds (3/11; 27.3\%),

All Rights Reserved (C) 2015 Universidad Nacional Autónoma de México, Instituto de Biología. This is an open access item distributed under the

\footnotetext{
* Autora para correspondencia.

Correo electrónico: bianque01@yahoo.com.br (J. Bianque-de Oliveira).

La revisión por pares es responsabilidad de la Universidad Nacional Autónoma de México.
}

grupos de parásitos más comunes y el daño que ocasionan al huésped se asocia con su patogenicidad, intensidad de la infección y el estado inmune del hospedador (Godoy, 2006).

Amazona amazonica (L.) (Psittaciformes, Psittacidae), conocidos como Lora Amazónica o Loro Real, viven en grupos. Tienen un área de distribución amplia en América del Sur y se alimentan principalmente de semillas y frutos (Moura, 2007; Piratelli, Andrade y Lima-Filho, 2005). En las últimas décadas se ha detectado una disminución de su población, debido principalmente a la pérdida de hábitat y a la caza para su comercialización como mascotas (Marini y Garcia, 2005), aunque está clasificada 
en la categoría de «poco preocupante» por la Unión Internacional para la Conservación de la Naturaleza (IUCN, 2014). Uno de los aspectos más importantes para implementar medidas en la conservación de especies es conocer las interacciones que establecen con los diferentes grupos de parásitos en su ambiente natural, ya que esto puede evitar el surgimiento y/o incremento de enfermedades, así como proporcionar información sobre el estado de un ecosistema en particular. Por lo anterior, el objetivo de este estudio fue identificar los parásitos gastrointestinales de A. amazonica en vida libre en el estado de Pernambuco, nordeste de Brasil.

En el primer semestre del 2013, ejemplares de A. amazonica de un área protegida en Barra de Guabiraba, estado de Pernambuco ( $8^{\circ} 28^{\prime} 41^{\prime}$ S; 35 39'1” O/571 m) fueron trasladados del Instituto Brasileiro de Meio Ambiente e dos Recursos Naturais Renováveis (IBAMA) a la Floresta Nacional do Araripe, estado de Ceará. Durante el traslado, 19 aves adultas murieron y fueron enviadas al Departamento de Biología de la Universidad Federal Rural de Pernambuco (UFRPE) (documento IBAMA, n. $\left.{ }^{\circ} 44 / 2013\right)$. Los helmintos recolectados en los tubos digestivos de estas aves fueron enviados al Laboratorio de Parasitología (LAPAR) de la UFRPE para su identificación. Los nematodos fueron fijados en AFA, conservados en etanol $70{ }^{\circ} \mathrm{GL}$ y clarificados en lactofenol (Monteiro, Amato y Amato, 2006). Los cestodos se tiñeron con hematoxilina y fueron aclarados con fenol, según la metodología adaptada de Amato, Böeger y Amato (1991) y Hoffmann (1987). Los helmintos fueron montados en portaobjetos con resina sintética permanente (Entellan®), identificados de acuerdo con las claves de Anderson, Chabaud y Willmott (2009), Khalil, Jones y Bray (1994) y Vicente, Rodrigues, Gomes y Pinto (1995) e incorporados a la colección del LAPAR/UFRPE (75-78). La prevalencia de infección fue calculada de acuerdo con Bush, Lafferty, Lotz y Shostak (1997).

De las 19 aves estudiadas, $11(57.9 \%)$ se encontraron parasitadas. De estas, $8(8 / 11 ; 72.7 \%)$ estuvieron infectadas por el nematodo Ascaridia hermaphrodita (Froelich, 1789) (Ascaridoidea, Ascaridiidae) y 3 (27.3\%) por el cestodo Raillietina sp. (Cyclophyllidea, Davaineidae). Ambos parásitos son registrados por primera vez parasitando a A. amazonica en el estado de Pernambuco. Infecciones por Ascaridia son muy comunes en aves y representan un riesgo para su salud; su presencia está asociada con signos clínicos como pérdida de peso, anorexia y muerte en casos severos (Godoy, 2006). González-Acuña, Fabry, Nascimento y Tebaldi (2007) y Melo et al. (2013) demostraron que la obstrucción intestinal debido a la infección masiva por $A$. hermaphrodita es una de las causas de mortalidad en psitácidos. Además de la intensidad de la infección, el estado inmunológico de las aves también puede contribuir a los daños producidos por los parásitos (Godoy, 2006).

En Brasil, Melo et al. (2013) registraron el parasitismo por Raillietina sp. en psitácidos de la especie A. aestiva en cautiverio. Aunque los cestodos sean frecuentes en las aves silvestres, es inusual su asociación con mortalidad. En general, las aves parasitadas pueden mostrar anemia, pérdida de peso y diarrea, pero las infecciones más graves pueden facilitar la aparición de otras enfermedades que pueden causar la muerte del animal (Doneley, 2009; Godoy, 2006).

El hábito gregario de $A$. amazonica puede representar un riesgo para poblaciones afectadas por infecciones parasitarias; la alta densidad poblacional de los hospederos puede ser un factor influyente en la mortalidad de individuos que enfrentan una enfermedad (Hochachka y Dhondt, 2000). Los hábitos de alimentación de las aves también pueden estar relacionados con el parasitismo. Las aves que se alimentan de frutas y granos, como es el caso de A. amazonica, tienen grandes posibilidades de contaminación durante la búsqueda de alimento, al ingerir fauna de acompañamiento (Costa et al., 2010), y a su vez, pueden dispersar patógenos durante el forrajeo (Barrera-Guzmán y Guillén-Hernández, 2008).

De acuerdo con Costa et al. (2010), el análisis parasitológico en estas especies constituye una herramienta importante para la evaluación de las condiciones sanitarias del ambiente. A pesar de la limitación de las investigaciones en este campo por la dificultad para acceder a los hospedadores, el conocimiento sobre las enfermedades y sus implicaciones es de fundamental importancia para los programa de manejo y conservación de la biodiversidad, especialmente en países megadiversos como Brasil.

\section{Referencias}

Amato, J. F. R., Böeger, W. A. y Amato, S. B. (1991). Protocolos para laboratório: coleta e processamento de parasitos de pescado. Seropédica: Gráfica da Universidade Federal Rural do Rio de Janeiro.

Anderson, R. C., Chabaud, A. G. y Willmott, S. (2009). Keys to the nematode parasites of vertebrates. Archival volume. Wallingford: CAB International.

Barrera-Guzmán, A. O. y Guillén-Hernández, S. (2008). Helmintos intestinales en aves Ciconiiformes de la ciénaga de Chuburná, Yucatán, México. Revista Mexicana de Biodiversidad, 79, 525-527.

Bush, A. O., Lafferty, K. D., Lotz, J. M. y Shostak, A. W. (1997). Parasitology meets ecology in its own terms: Margolis et al. revisited. Journal of Parasitology, 83, 575-583.

Catão-Dias, J. L. (2003). Doenças e seus impactos sobre a biodiversidade. Ciência e Cultura, 55, 32-34.

Costa, I. A., Coelho, C. D., Bueno, C., Ferreira, I. y Freire, E. (2010). Ocorrência de parasitos gastrintestinais em aves silvestres no município de Seropédica, Rio de Janeiro, Brasil. Ciência Animal Brasileira, 11, 914-922.

Doneley, R. J. T. (2009). Bacterial and parasitic diseases of parrots. Veterinay Clinical of Exotic Animals, 12, 417-432.

Godoy, S. N. (2006). Psittaciformes (Arara, Papagaio, Periquito). En Z. S. Cubas, J. C. R. Silva, y J. L. C. Dias (Eds.), Tratado de Animais Selvagens (pp. 222-251). São Paulo: Roca.

González-Acuña, D., Fabry, M., Nascimento, A. A. y Tebaldi, J. H. (2007). Death of two slender-billed parakeet (King) (Enicognathus leptorhynchus) (aves, Psittacidae) by Ascaridia hermaphrodita (Froelich, 1789, Railliet and Henry, 1914) at the National Zoo of Santiago, Chile. Arquivo Brasileiro de Medicina Veterinária e Zootecnia, 59, 539-540.

Hochachka, W. M. y Dhondt, A. A. (2000). Density-dependent decline of host abundance resulting from a new infectious disease. Proceedings of the National Academy of Sciences, 97, 5303-5306.

Hoffmann, R. P. (1987). Diagnóstico de parasitismo veterinário. Sulina: Porto Alegre.

IUCN (International Union for Conservation of Nature). (2014). Red list of threatened species. Version 2014.2. Recuperado el 16 septiembre 2014 de www.iucnredlist.org

Khalil, L. F., Jones, A. y Bray, R. A. (1994). Keys to the Cestode parasites of vertebrates. Wallingford: CAB International. 
Marini, M. A. y Garcia, F. I. (2005). Conservação de aves no Brasil. Megadiversidade, 1, 95-102.

Melo, C. M. F., Oliveira, J. B., Feitosa, T. F., Vilela, V. L. R., Athayde, A. C. R., Dantas, A. F. M., et al. (2013). Parasites of Psittaciformes and Accipitriformes in Paraiba State, Northeastern Brazil. Revista Brasileira de Parasitologia Veterinária, 22, 314-317.

Monteiro, C. M., Amato, J. F. R. y Amato, S. B. (2006). Primeiro registro de Syncuaria squamata (Linstow) (Nematoda, Acuariidae) em biguás, Phalacrocorax brasilianus (Gmelin) (aves Phalacrocoracidae) no Brasil. Revista Brasileira de Zoologia, 23, 1268-1272.
Moura, L. N. D. (2007). Comportamento do Papagaio-do-mangue Amazona amazonica: gregarismo, ciclos nictemerais e comunicação sonora. Tesis de maestría. Pará: Universidade Federal do Pará.

Piratelli, A., Andrade, V. A. y Lima-Filho, M. (2005). Aves de fragmentos florestais em área de cultivo de cana-de-açúcar no sudeste do Brasil. Iheringia, série Zoologia, 95, 217-222.

Vicente, J. J., Rodrigues, H. D. O., Gomes, D. C. y Pinto, R. M. (1995). Nematóides do Brasil. Parte IV: Nematóides de aves. Revista Brasileira de Zoologia, $12,1-273$. 